\title{
Magnetoelastic domains and magnetic field-induced strains in ferromagnetic shape memory alloys by phase-field simulation
}

\author{
L. J. Li, ${ }^{1}$ J. Y. Li, $\left.{ }^{1}, \mathrm{a}\right)$ Y. C. Shu, ${ }^{2}$ H. Z. Chen, ${ }^{2}$ and J. H. Yen ${ }^{2}$ \\ ${ }^{1}$ Department of Mechanical Engineering, University of Washington Seattle, Washington 98195-2600, USA \\ ${ }^{2}$ Institute of Applied Mechanics, National Taiwan University, Taipei 106, Taiwan, Republic of China
}

(Received 14 January 2008; accepted 10 April 2008; published online 29 April 2008)

\begin{abstract}
Magnetoelastic domains in ferromagnetic shape memory alloys evolve through either variant rearrangement or magnetization rotation, resulting in a large or a small magnetic field-induced strain depending on the magnitude of applied compressive stress. These phenomena are simulated in this letter using an unconventional phase-field model motivated by energy-minimizing multirank laminated domain structures. The results agree well with experiments, and confirm the analysis of Ma and Li [Appl. Phys. Lett. 90, 172504 (2007)] based on an energy minimization theory. (C) 2008 American Institute of Physics. [DOI: 10.1063/1.2918127]
\end{abstract}

Ferromagnetic shape memory alloys (FSMAs) possess both ferroelastic and ferromagnetic ordering simultaneously, ${ }^{1}$ and this magnetoelastic coupling makes it possible to manipulate the ferroelastic domains of FSMA by magnetic field. The resulted variant rearrangement leads to a magnetic fieldinduced strain as high as $10 \%,{ }^{2-5}$ which is very attractive for actuator applications. However, the blocking stress of FSMA is relatively small, and the magnetic field-induced strain dramatically drops when the external compressive stress increases, ${ }^{5-7}$ which seriously limits FSMA's applications as actuators. Using an energy-minimization theory, $\mathrm{Ma}$ and $\mathrm{Li}^{7}$ suggested that when the external compressive stress exceeds a critical threshold, the magnetic field-induced variant rearrangement in FSMA will be frozen, and magnetization rotation will take over instead as the dominant mechanism for domain structure evolution, leading to a magnetostrictive strain that is orders of magnitude smaller. Understanding the formation and evolution of FSMA domain structure thus is not only important from the scientific interests but is also critical to the applications of FSMA.

While a few theoretical models have been developed for FSMA, ${ }^{8-19}$ direct numerical simulation of magnetoelastic domains has been rarely attempted. ${ }^{20,21}$ Capturing variant rearrangement and magnetization rotation under a unified theoretical framework is particularly challenging. In this letter, we report an unconventional phase-field simulation of FSMA to accomplish that. To demonstrate this, we consider a tetragonal FSMA. It has three ferroelastic variants, and each of them is distorted by a transformation strain $\boldsymbol{\varepsilon}^{(i)}$ and is magnetized by a saturation magnetization $M_{s}$ that prefers to be aligned along the easy axis $\mathbf{r}^{(i)}$ of the respective variants, with

$$
\begin{aligned}
& \boldsymbol{\varepsilon}^{(i)}=\alpha \mathbf{I}-(\alpha-\beta) \mathbf{r}^{(i)} \otimes \mathbf{r}^{(i)}, \\
& \mathbf{r}^{(1)}=(1,0,0), \quad \mathbf{r}^{(2)}=(0,1,0), \quad \mathbf{r}^{(3)}=(0,0,1),
\end{aligned}
$$

where $\alpha$ and $\beta$ are material parameters, and $\mathbf{I}$ is the unit second rank tensor. Notice that the easy axis of magnetization is intimately coupled with the transformation strain. Nevertheless, the direction of magnetization $\mathbf{M}=M_{s} \mathbf{m}$ in

\footnotetext{
${ }^{\text {a) }}$ Author to whom correspondence should be addressed. Electronic mail:
} jjli@u.washington.edu. each variant is not constrained to the easy axis, and can rotate away from the easy axis with an energy penalty, resulting in a magnetostrictive strain $\boldsymbol{\varepsilon}^{m}(\mathbf{m})$ that is orders of magnitude smaller than transformation strain. ${ }^{22}$ As such, both strain and magnetization are needed to describe the domain structure of FSMA.

It is well known that phase-changing materials such as FSMA form very characteristic domain structures consisting of multiple variants to reduce the overall energy of the system. While transformation strains are used as order parameters in the conventional phase-field simulation of ferroelastic domain structures, ${ }^{21,23}$ Shu and $\mathrm{Yen}^{24}$ and Shu et al. ${ }^{25}$ suggested that the local volume fractions of variants can be used as field variables instead. For a three-variant system such as tetragonal FSMA, the local transformation strain is related to $\gamma_{i}$, the volume fraction of variant $i$, as follows:

$$
\boldsymbol{\varepsilon}^{*}=\gamma_{1} \boldsymbol{\varepsilon}^{(1)}+\gamma_{2} \boldsymbol{\varepsilon}^{(2)}+\gamma_{3} \boldsymbol{\varepsilon}^{(3)} \text {. }
$$

Obviously, only two of the $\gamma_{i}$ are independent, and $\mu_{1}$ and $\mu_{2}$ are introduced to reflect this constraint, with

$$
\gamma_{1}=\mu_{1}, \quad \gamma_{2}=\left(1-\mu_{1}\right) \mu_{2}, \quad \gamma_{3}=\left(1-\mu_{1}\right)\left(1-\mu_{2}\right) .
$$

This representation is motivated by the multirank laminated domain configuration, which is energy-minimizing when $\boldsymbol{\varepsilon}^{(i)}$ are pariwise compatible. ${ }^{12,26,27}$ Through Eqs. (2) and (3), the equivalence of transformation strain $\boldsymbol{\varepsilon}^{*}$ and $\boldsymbol{\mu}=\left(\mu_{1}, \mu_{2}\right)$ is established.

When $\boldsymbol{\mu}$ is used as field variables instead of $\boldsymbol{\varepsilon}^{*}$, the potential energy of a FSMA occupying a domain $\Omega$ can be expressed as ${ }^{7,22}$

$$
\begin{aligned}
\mathcal{I}(\boldsymbol{\mu}, \mathbf{m})= & \int_{\Omega}\left\{W^{\mathrm{int}}+W_{s}^{\mathrm{ani}}+W_{m}^{\mathrm{ani}}+W^{\mathrm{elas}}-\boldsymbol{\sigma}^{0} \cdot \boldsymbol{\varepsilon}\right. \\
& \left.-\mu_{0} \mathbf{H}^{0} \cdot \mathbf{M}\right\} d \mathbf{x}+\frac{\mu_{0}}{2} \int_{\mathrm{R}^{3}}|\nabla \phi|^{2} d \mathbf{x},
\end{aligned}
$$

where $W^{\text {int }}=A_{1}|\nabla \boldsymbol{\mu}|^{2}+A_{2}|\nabla \mathbf{m}|^{2}$ penalizes gradient of internal variables and thus leads to interfacial energy across magnetoelastic domain walls, $W_{s}^{\text {ani }}=K_{s} \Sigma_{i=1}^{2} \mu_{i}^{2}\left(1-\mu_{i}\right)^{2}$ is the anisotropy energy that penalizes the deviation of transformation strain from the ground state, which allows us to express energy wells of FSMA explicitly instead of through expansion of polynomial of transformation strain, and thus simpli- 
fies the model development considerably, $W_{m}^{\mathrm{ani}}=K_{u}(1$ $\left.-\left[\mathbf{m} \cdot \mathbf{r}^{*}(\boldsymbol{\mu})\right]^{2}\right)$ is the magnetic anisotropy energy that penalizes the magnetization rotation away from the easy axis, given by $\mathbf{r}^{*}=\gamma_{1} \mathbf{r}^{(1)}+\gamma_{2} \mathbf{r}^{(2)}+\gamma_{3} \mathbf{r}^{(3)}$, which explicitly couples the ferroelastic ordering $\boldsymbol{\mu}$ and ferromagnetic ordering $\mathbf{m}$. Moreover, $W^{\text {elas }}=\frac{1}{2}\left[\boldsymbol{\varepsilon}-\boldsymbol{\varepsilon}^{*}(\boldsymbol{\mu})-\boldsymbol{\varepsilon}^{m}(\mathbf{m})\right] \cdot \mathbf{C}\left[\boldsymbol{\varepsilon}-\boldsymbol{\varepsilon}^{*}(\boldsymbol{\mu})\right.$ $\left.-\boldsymbol{\varepsilon}^{m}(\mathbf{m})\right]$ is the elastic energy resulting from the incompatibility of transformation and magnetostrictive strains, which can be determined by solving mechanical equilibrium equation, ${ }^{24}$ where $\boldsymbol{\varepsilon}$ is the total strain and $\mathbf{C}$ is the elastic stiffness. The last two terms in the first integral are potential energies associated with the applied magnetic field $\mathbf{H}^{0}$ and stress $\boldsymbol{\sigma}^{0}$, and the last integral is the demagnetization energy due to the magnetization distribution in FSMA, which can be determined by solving Maxwell's equation in full space, ${ }^{7}$ where $\phi$ is the magnetic potential and $\mu_{0}$ is the permeability of free space.

Under an external magnetic or mechanical loading, both $\boldsymbol{\mu}$ and $\mathbf{m}$ will evolve to minimize the potential energy $\mathcal{I}$ in Eq. (4). The evolution of $\mu$ is given by

$$
\frac{\partial \boldsymbol{\mu}}{\partial t}=-L \frac{\delta \mathcal{I}}{\delta \boldsymbol{\mu}}=L\left[\mathbf{F}^{\mathrm{int}}+\mathbf{F}_{s}^{\mathrm{ani}}+\mathbf{F}_{m}^{\mathrm{ani}}+\mathbf{F}^{\text {elas }}\right]
$$

which governs the variant rearrangement process. In Eq. (5), $L$ is the mobility constant, $\mathbf{F}^{\text {int }}=2 A_{1} \nabla^{2} \boldsymbol{\mu}$ drives the coarsening of ferroelastic domains, $\mathbf{F}_{s}^{\mathrm{ani}}=-\partial / \partial \boldsymbol{\mu} W_{s}^{\mathrm{ani}}(\boldsymbol{\mu})$ and $\mathbf{F}_{m}^{\text {ani }}=-\partial / \partial \boldsymbol{\mu} W_{m}^{\text {ani }}(\boldsymbol{\mu}, \mathbf{m})$ select the particular set of variants, with the second one coupling the transformation strain and the magnetization, and $\mathbf{F}^{\text {elas }}=\mathbf{C}\left[\boldsymbol{\varepsilon}-\boldsymbol{\varepsilon}^{*}(\boldsymbol{\mu})\right.$ $\left.-\boldsymbol{\varepsilon}^{m}(\mathbf{m})\right] \cdot \partial \boldsymbol{\varepsilon}^{*}(\boldsymbol{\mu}) / \partial \boldsymbol{\mu}$ drives the refining of ferroelastic domains. Notice that $\mathbf{C}\left[\boldsymbol{\varepsilon}-\boldsymbol{\varepsilon}^{*}(\boldsymbol{\mu})-\boldsymbol{\varepsilon}^{m}(\mathbf{m})\right]$ is actually the stress in FSAM that is related to applied stress at boundary. ${ }^{22}$ On the other hand, the evolution of magnetization is given by Landau-Lifschitz-Gilbert equation

$$
\frac{\partial \mathbf{m}}{\partial t}=-\gamma_{g} \mathbf{m} \times \mathbf{H}^{\mathrm{eff}}-\delta \gamma_{g} \mathbf{m} \times\left(\mathbf{m} \times \mathbf{H}^{\mathrm{eff}}\right),
$$

which governs the process of magnetization rotation, where $\gamma_{g} \approx 2.21 \times 10^{5} \mathrm{~m} /(\mathrm{A} \mathrm{s})$ is the gyromagnetic ratio, $\delta$ is the dimensionless damping coefficient, and the effective magnetic field is given by

$$
\mathbf{H}^{\mathrm{eff}}=-\frac{1}{\mu_{0} M_{s}} \frac{\delta \mathcal{I}}{\delta \mathbf{m}}=\mathbf{H}^{e}+\mathbf{H}^{a}+\mathbf{H}^{s}+\mathbf{H}^{0}+\mathbf{H}^{d},
$$

where $\mathbf{H}^{e}=2 A_{2} / \mu_{0} M_{s} \nabla^{2} \mathbf{m}$ drives the coarsening of magnetic domains, $\mathbf{H}^{a}=-1 / \mu_{0} M_{s} \partial / \partial \mathbf{m} W_{m}^{\text {ani }}(\boldsymbol{\mu}, \mathbf{m})$ selects preferred magnetization direction, $\mathbf{H}^{s}=1 / \mu_{0} M_{s} \mathbf{C}\left[\boldsymbol{\varepsilon}-\boldsymbol{\varepsilon}^{*}(\boldsymbol{\mu})\right.$ $\left.-\boldsymbol{\varepsilon}^{m}(\mathbf{m})\right] \cdot \partial \boldsymbol{\varepsilon}^{m}(\mathbf{m}) / \partial \mathbf{m}$ couples the magnetization and transformation and magnetostrictive strains, and $\mathbf{H}^{d}=-\nabla \phi$ is the demagnetization field. Thus, framework is established to study the formation and evolution of magnetoelastic domains in FSMA in terms of $\boldsymbol{\mu}$ and $\mathbf{m}$.

A two-dimensional simulation of FSMA $\mathrm{Ni}_{2} \mathrm{MnGa}$ is implemented by solving Eqs. (5) and (6) under the periodic boundary condition (BC), with the following material constants: : $7,11,16 K_{s}=K_{u}=1.65 \times 10^{5} \mathrm{~J} / \mathrm{m}^{3}, \quad A_{1} /\left(K_{s} l_{0}^{2}\right)$ $=A_{2} /\left(K_{u} l_{0}^{2}\right)=10^{-4}$, with $l_{0}$ being the length of discretization, $\alpha=0.021, \beta=-0.034$, Young's modulus is taken to be $154 \mathrm{GPa}$, and Poisson's ratio is assumed to be 0.3. The demagnetization field consists of fields due to shape anisotropy and internal magnetization incompatibility. The shape anisoDownloaded 21 Dec 2008 to 140.112 .113 .225 . Redistribution subje (a)
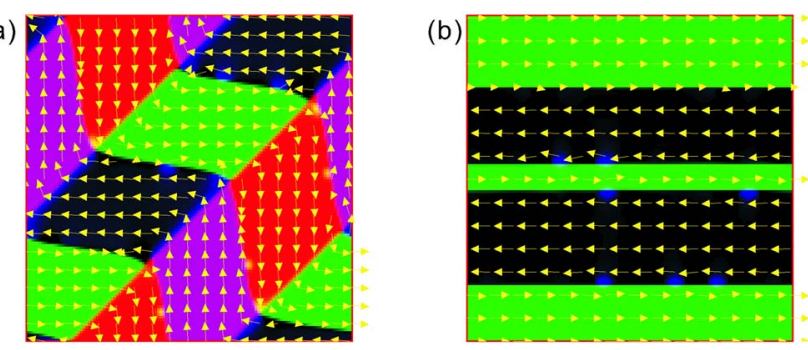

FIG. 1. (Color online) Formation of magnetoelastic domains: (a) Rank-2 domain pattern in FSMA under clamped BC and (b) rank-1 domain pattern in FSMA under compressive stress. Green and black colors indicate variant 1, while fuchsia and red indicate variant 3; arrow indicates the magnetization direction.

tropy part is evaluated using demagnetization factors $N_{1}$ $=0.194, N_{2}=N_{3}=0.403$, which are calculated from the dimensions of $9 \times 5 \times 5 \mathrm{~mm}^{3}$ rod, while the field arising from internal incompatibility is calculated in Fourier space under the periodic boundary condition. ${ }^{28}$ Since variant 2 is favored by neither the applied stress nor magnetic field, it is excluded from consideration, and a two-dimensional simulation will be sufficient to capture the essence of the problem. We first consider the formation of magnetoelastic domain structure in FSMA subjected to clamped $\mathrm{BC}$ and random initial condition, and a self-accommodating domain structure emerges, as shown in Fig. 1(a) in a $128 \times 128$ cell, in which each arrow actually spans multiple cells to make the illustration clear, otherwise the figure will become too crowded. Increasing the computational size further does not change the simulation results. Note that the domain structure consists of two ferroelastic variants 1 and 3 of equal volume fraction, separated

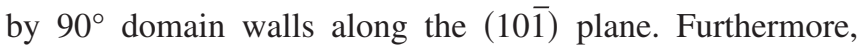
each of the ferroelastic domain is divided into two different kinds of magnetic domains, separated by $180^{\circ}$ domain walls along the (100) or (001) plane, and the combined magnetoelastic domains are, indeed, a rank-2 laminate as predicted by the constrained theory. ${ }^{12}$ On the other hand, when a compressive stress is applied along the [100] axis, a single ferroelastic variant 1 consists of two magnetic domains, separated by $180^{\circ}$ domain walls, emerges as a rank- 1 laminate as also predicted by the constrained theory, ${ }^{12}$ as shown in Fig. 1(b).

We then consider a $\mathrm{Ni}_{2} \mathrm{MnGa}$ rod subjected to a fixed compress stress $\boldsymbol{\sigma}^{0}$ along the longer axis of the rod, parallel to the [100] axis of the crystal, and a varying magnetic field $\mathbf{H}^{0}$ parallel to the [001] axis, a typical experimental configuration of FSMA. ${ }^{5}$ A rank-1 laminate with equal volume fraction of $180^{\circ}$ magnetic domains similar to Fig. 1(b) is used as the initial configuration but with a single layer of variant 3 less than $1 \%$ of total volume added to facilitate nucleation. As the magnetic field along the [001] axis increases, the domain structure of FSMA will evolve, through either variant rearrangement or magnetization rotation, depending on the magnitude of the applied compress stress, as suggested by $\mathrm{Ma}$ and $\mathrm{Li}^{7}{ }^{7}$ This is, indeed, observed in our simulation, as shown in Figs. 2 and 3. For example, when a small compressive stress of $0.6 \mathrm{MPa}$ is applied, small magnetization rotation occurs first, but variant rearrangement quickly takes over as the dominant evolution mechanism, leading to a large jump in magnetic field-induced strain at $0.3 \mathrm{~T}$ and, correspondingly, a larger slope of magnetization curve, as shown by the solid green curve in Fig. 2, which agrees very well to AIP license or copyright; see http://apl.aip.org/apl/copyright.jsp 

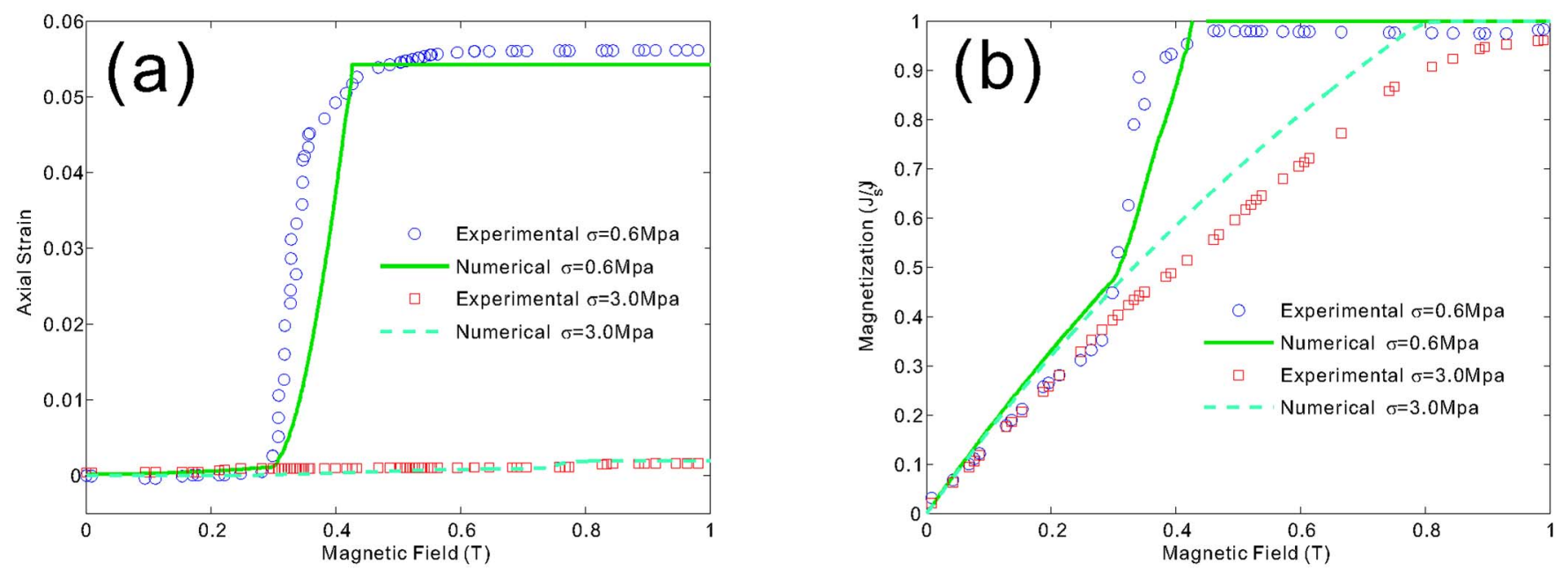

FIG. 2. (Color online) Axial strain (a) and magnetization (b) vs the applied magnetic field under compressive stress of 0.6 and $3 \mathrm{MPa}$, respectively.

with experimental data. ${ }^{5} \mathrm{~A}$ closer examination of the intermediate domain structure before the saturation of magnetization reveals a very characteristic rank-2 domain structure, as shown in Fig. 3(a), where variant 3 is observed to grow at the expense of variant 1 , which is separated by a $90^{\circ}$ domain wall along the (101) plane. Small magnetization also exists in variant 1 , which is consistent with the analysis of Ma and $\mathrm{Li}^{7}{ }^{7}$ On the other hand, when a relatively large compress stress of $3 \mathrm{MPa}$ is applied, the variant rearrangement process is completely blocked, leading to magnetostrictive strain that is orders of magnitude smaller than transformation strain, as shown by the broken blue curve in Fig. 2(a), also in excellent agreement with experiment. ${ }^{5}$ No change in the slope of magnetization curve is observed before saturation, another indication of magnetization rotation instead of variant rearrangement which is again in good agreement with experiment. ${ }^{5}$ Examination of the intermediate domain structure before magnetization saturation reveals that the ferroelastic variant is indeed unchanged throughout the magnetization process even with the presence of prescribed nucleation layer. Only magnetization rotation occurs, as shown in Fig. 3(b), where a rank-1 laminate is observed with identical ferroelastic variant but different magnetization directions. Simulation on an intermediate stress of $1.4 \mathrm{MPa}$ has also been carried out in good agreement with experiment. In all these simulations, all the conditions are kept identical except the magnitude of the applied compressive stress.

In summary, we have developed an unconventional phase-field model to simulate the formation and evolution of magnetoelastic domains and magnetic field-induced strain in
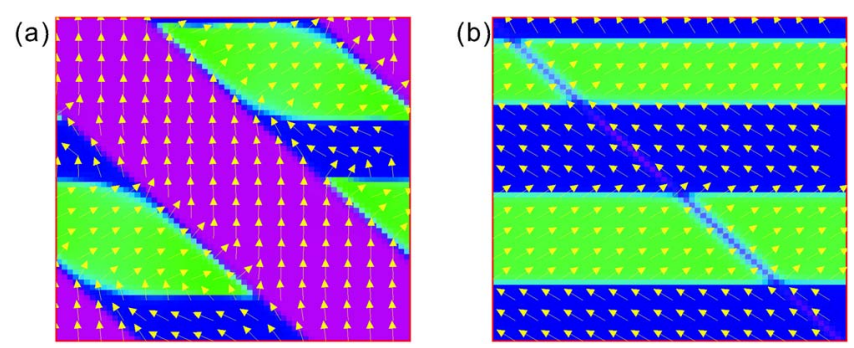

FIG. 3. (Color online) Magnetoelastic domains at intermediate stages of magnetization under different compressive stresses of $0.6 \mathrm{MPa}$ (a) and 3.0 MPa (b). Green and blue colors indicate variant 1 , while fuchsia indicates variant 3; arrow is used to indicate the magnetization direction.
FSMA, where both variant rearrangement and magnetization rotation are captured. The simulation agrees well with experiments, and confirms the analysis of $\mathrm{Ma}$ and $\mathrm{Li}^{7}$ that variant rearrangement in FSMA is blocked at large compressive stress, resulting in much reduced magnetic field-induced strain in FSMA.

We acknowledge the financial support from US ARO (W911NF-07-1-0410) and AFOSR (FA9550-07-1-0175). Y.C.S. also acknowledges the support of TW NSC Grant (NSC-96-2221-E-002-014).

${ }^{1}$ K. Ullakko, J. K. Huang, C. Kantner, R. C. O'Handley, and V. V. Kokorin, Appl. Phys. Lett. 69, 1966 (1996).

${ }^{2}$ R. Tickle and R. D. James, J. Magn. Magn. Mater. 195, 627 (1999).

${ }^{3}$ S. J. Murray, M. Marioni, S. M. Allen, R. C. O’Handley, and T. A. Lograsso, Appl. Phys. Lett. 77, 886 (2000).

${ }^{4}$ A. Sozinov, A. A. Likhachev, N. Lanska, and K. Ullakko, Appl. Phys. Lett. 80, 1746 (2002)

${ }^{5}$ O. Heczko, J. Magn. Magn. Mater. 290, 787 (2005).

${ }^{6}$ O. Heczko, L. Straka, and S. P. Hannula, Mater. Sci. Eng., A 438, 1003 (2006).

${ }^{7}$ Y. F. Ma and J. Y. Li, Appl. Phys. Lett. 90, 172504 (2007).

${ }^{8}$ R. D. James and M. Wuttig, Philos. Mag. A 77, 1273 (1998).

${ }^{9}$ R. C. O'Handley, J. Appl. Phys. 83, 3263 (1998).

${ }^{10}$ V. A. L'vov, E. V. Gomonaj, and V. A. Chernenko, J. Phys.: Condens. Matter 10, 4587 (1998).

${ }^{11}$ A. A. Likhachev and K. Ullakko, Phys. Lett. A 275, 142 (2000).

${ }^{12}$ A. DeSimone and R. D. James, J. Mech. Phys. Solids 50, 283 (2002).

${ }^{13}$ P. Mullner, V. A. Chernenko, M. Wollgarten, and G. Kostorz, J. Appl. Phys. 92, 6708 (2002).

${ }^{14}$ L. Hirsinger and C. Lexcellent, J. Phys. IV 112, 977 (2003).

${ }^{15}$ A. A. Likhachev, A. Sozinov, and K. Ullakko, Mater. Sci. Eng., A 378, 513 (2004)

${ }^{16}$ J. Kiang and L. Tong, J. Magn. Magn. Mater. 292, 394 (2005).

${ }^{17}$ B. Kiefer and D. C. Lagoudas, Philos. Mag. 85, 4289 (2005).

${ }^{18}$ D. I. Paul, R. C. O'Handley, and B. Peterson, J. Appl. Phys. 97, 10M312 (2005).

${ }^{19}$ Y. F. Ma and J. Y. Li, Acta Mater. 55, 3261 (2007).

${ }^{20}$ T. Koyama and H. Onodera, Mater. Trans. 44, 2503 (2003).

${ }^{21}$ J. X. Zhang and L. Q. Chen, Philos. Mag. Lett. 85, 533 (2005).

${ }^{22}$ Y. C. Shu, M. P. Lin, and K. C. Wu, Mech. Mater. 36, 975 (2004).

${ }^{23}$ L. Q. Chen, Annu. Rev. Mater. Res. 32, 113 (2002).

${ }^{24}$ Y. C. Shu and J. H. Yen, Appl. Phys. Lett. 91, 021908 (2007).

${ }^{25}$ Y. C. Shu, J. H. Yen, H. Z. Chen, J. Y. Li, and L. J. Li, Appl. Phys. Lett. 92, 052909 (2008).

${ }^{26}$ K. Bhattacharya, Continuum Mech. Thermodyn. 5, 205 (1993).

${ }^{27}$ J. Y. Li and D. Liu, J. Mech. Phys. Solids 52, 1719 (2004).

${ }^{28}$ J. X. Zhang and L. Q. Chen, Acta Mater. 53, 2845 (2005). 